\title{
REGULARIZING EFFECT OF THE INTERPLAY BETWEEN COEFFICIENTS IN DIRICHLET PROBLEMS WITH CONVECTION OR DRIFT TERMS
}

\section{LUCIO BOCCARDO*}

\begin{abstract}
There are very important results by Enrique Zuazua on the subject of the convectiondiffusion equation

$$
u_{t}-\operatorname{div}(a(x) \nabla u)=-d \cdot \nabla\left(|u|^{q-1} u\right), \quad \text { in }(0,+\infty) \times \mathbb{R}^{N}
$$

In some sense this paper deals with a linear (i.e. $q=1)$ elliptic counterpart of the above equation if $d$ is not constant.

We prove regularizing results on the solutions, under assumptions of interplay between the datum and the coefficient of the zero order term or between the modulus of the drift and the coefficient of the zero order term.
\end{abstract}

Mathematics Subject Classification. 35J15, 35J60.

Received November 17, 2020. Accepted February 2, 2021.

Enrike, but this is how Paris 6 was in the early days when we were very poor, very happy and very young (not sixty-year-old)

\section{INTRODUCTION}

There are very important results by Enrique Zuazua on the subject of convection-diffusion equation of the type

$$
u_{t}-\operatorname{div}(a(x) \nabla u)=-d \cdot \nabla\left(|u|^{q-1} u\right), \quad \text { in }(0,+\infty) \times \mathbb{R}^{N}
$$

where $d \in \mathbb{R}^{N}$ and $a(x)$ is a matrix satisfying suitable ellipticity assumptions (see [19-21]).

In some sense this paper deals with a linear (i.e. $q=1$ ) elliptic counterpart of the above equation if $d$ is not constant.

Keywords and phrases: Regularizing effect, interplay between coefficients, problems with convection terms, problems with drift terms.

Sapienza Università di Roma - Istituto Lombardo, Roma, Italy.

* Corresponding author: boccardo@mat.uniroma1.it 


\subsection{Setting}

This paper is concerned with Dirichlet problems with lower order terms depending on the gradient of the solution (convection type of drift type); in particular, we prove regularizing results on the solutions, under assumptions of interplay between the datum and the coefficient of the zero order term or between the modulus of the drift and the coefficient of the zero order term.

To be more precise, now, we briefly introduce

- Dirichlet problems with lower order terms depending on the gradient of the solution (convection type of drift type);

- regularizing effects on the solutions of Diriclet problems, under assumptions of interplay between coefficients.

Let $\Omega$ be a bounded, open subset of $\mathbb{R}^{N}, N>2$, and $M: \Omega \rightarrow \mathbb{R}^{N^{2}}$, be a bounded and measurable matrix such that

$$
\alpha|\xi|^{2} \leq M(x) \xi \cdot \xi, \quad|M(x)| \leq \beta, \quad \text { a.e. } x \in \Omega, \quad \forall \xi \in \mathbb{R}^{N}
$$

We assume that $E(x)$ is a vector field and $a(x), f(x)$ are functions such that

$$
\begin{gathered}
E \in\left(L^{N}(\Omega)\right)^{N}, \\
f \in L^{m}(\Omega), \quad m \geq 1, \\
0 \leq a(x) \in L^{1}(\Omega),
\end{gathered}
$$

and we consider the following boundary value problems: with a convection term

$$
\left\{\begin{aligned}
-\operatorname{div}(M(x) \nabla u)+a(x) u=-\operatorname{div}(u E(x))+f(x), & \text { in } \Omega ; \\
u=0, & \text { on } \partial \Omega ;
\end{aligned}\right.
$$

or with a drift term

$$
\left\{\begin{aligned}
-\operatorname{div}(M(x) \nabla \psi)+a(x) \psi=E(x) \cdot \nabla \psi+f(x), & \text { in } \Omega ; \\
\psi=0, & \text { on } \partial \Omega .
\end{aligned}\right.
$$

The two boundary problems above are linear, but it is easy to see that the differential operators may be not coercive, unless $\|E\|_{L^{N}}$ is sufficiently small with respect to $\alpha$ or $\operatorname{div}(E)=0$. It is worthy to recall the first papers about this subject: $[14,23]$.

Despite the coercitivity difficulty, in [5, 7] (see also [6]) a Stampacchia-Calderon-Zygmund theory is proved, with no restrictions on the norm of $E$ and no assumptions of the type $\operatorname{div}(E)=0$ :

A (Stampacchia theory) the boundary value problems (1.5) and (1.6) have a unique weak solution $\psi, u \in$ $W_{0}^{1,2}(\Omega) \cap L^{m^{* *}}(\Omega), m^{* *}=\frac{N m}{N-2 m}$, if $\frac{2 N}{N+2} \leq m<\frac{N}{2} ;$

B (Caldéron-Zygmund theory) the boundary value problems (1.5) and (1.6) have a distributional solution $u \in W_{0}^{1, m^{*}}(\Omega), m^{*}=\frac{N m}{N-m}$, if $1<m<\frac{2 N}{N+2} ;$

that is, the same results proved in [23] and [12] for the problems with $E=0$. The cases $m=1$ and $m>\frac{N}{2}$ are studied, but the results for $\psi$ and $u$ are slightly different. 
We also recall the contributions of the papers [8-11, 15-18].

A second important feature of this paper is the assumption of interplay between coefficients, introduced in [1] (and developed in [2,3]): consider the boundary value problems (1.5) (or (1.6), it is the same) with $E=0$, under the assumption

$$
\text { there exists } Q>0 \text { such that }|f(x)| \leq Q a(x) \in L^{1}(\Omega) \text {. }
$$

Then in [1] is proved that the boundary value problem

$$
\left\{\begin{aligned}
-\operatorname{div}(M(x) \nabla w)+a(x) w=f(x), & \text { in } \Omega ; \\
u=0, & \text { on } \partial \Omega ;
\end{aligned}\right.
$$

has a weak bounded (with the sharp estimate $|w(x)| \leq Q$ ) solution belonging to $W_{0}^{1,2}(\Omega)$, in spite of the meagre $L^{1}$ summability of the datum $f(x)$.

Here we consider the boundary value problems (1.5), (1.6) and we study the impact of (1.7) on the regularity of the solutions $u, \psi$. We point out some results proved in the following sections, under the assumptions (1.1), (1.2), (1.4) and (1.7).

- There exists a unique weak solution $u$ of (1.5), that is $u$ satisfying

$$
\left\{\begin{array}{l}
u \in W_{0}^{1,2}(\Omega): \\
\int_{\Omega} M(x) \nabla u \nabla \varphi+\int_{\Omega} a(x) u \varphi=\int_{\Omega} u E(x) \nabla \varphi+\int_{\Omega} f(x) \varphi(x), \\
\forall \varphi \in W_{0}^{1,2}(\Omega) \cap L^{\infty}(\Omega)
\end{array}\right.
$$

moreover $u$ belongs to $L^{\rho}(\Omega)$, for every $\rho \geq 1$ (Thm. 2.3).

- If $E \in\left(L^{\sigma}(\Omega)\right)^{N}, \sigma>N$ (instead of (1.2)), the weak solution $u$ of (1.8) belongs $L^{\infty}(\Omega)$ (Thm. 2.5).

- There exists a unique weak solution $\psi$ of (1.6) (Thm. 2.7),

that is

$$
\left\{\begin{array}{l}
\psi \in W_{0}^{1,2}(\Omega) \\
\int_{\Omega} M(x) \nabla \psi \nabla \varphi+\int_{\Omega} a(x) \psi \varphi=\int_{\Omega}[E(x) \nabla \psi] \varphi+\int_{\Omega} f(x) \varphi, \\
\forall \varphi \in W_{0}^{1,2}(\Omega) \cap L^{\infty}(\Omega),
\end{array}\right.
$$

We remark that the previous results improve those of the Stampacchia-Calderon-Zygmund theory A, B (above) and Examples 2.4 and 2.6 show the optimality of the results.

Furthermore, we consider a second interplay assumption

$$
\text { there exists } R>0 \text { such that }|E|^{2} \leq R a(x) \in L^{1}(\Omega) \text {, }
$$

which implies that $|E| \in L^{2}$ (a weaker assumption with respect to (1.2)) and we prove the existence

- of distributional solutions in $W_{0}^{1, q}(\Omega), q=\frac{N}{N-1}$ (Thm. 3.2), if $f \log (1+|f|) \in L^{1}(\Omega)$;

- of finite energy solutions (i.e. $u \in W_{0}^{1,2}(\Omega)$, Thm. 3.5), if $f \in L^{m}(\Omega)$ with $m \geq \frac{2 N}{N+2}$.

Even here, we remark the improvements with respect to the Stampacchia-Calderon-Zygmund theory A, B (above), thanks to the assumption (1.10).

Then (Thm. 3.7) we consider the impact of the assumptions (1.7) and (1.10) assumed jointly. 


\section{THE IMPACT OF INTERPLAY BETWEEN THE COEFFICIENT OF THE ZERO ORDER TERM AND THE DATUM}

The main assumption of this section is (1.7).

\subsection{Convection equation}

We repeat the nonlinear approach (following $[5,6]$ ) to the linear noncoercive problem (1.5), that we always understand in the weak form (1.8).

We set

$$
f_{n}(x)=\frac{f(x)}{1+\frac{1}{n}|f(x)|}, a_{n}(x)=\frac{a(x)}{1+\frac{Q}{n}|a(x)|}, E_{n}=\frac{E(x)}{1+\frac{R}{n}|E(x)|} .
$$

Note that, if $f \in L^{p}(\Omega), p \geq 1$, the Lebesgue theorem implies that the sequence $\left\{f_{n}\right\}$ strongly converges to $f$ in $L^{p}(\Omega)$.

We consider the weak solution $u_{n}$ of the approximated boundary value problems

$$
\left\{\begin{array}{l}
u_{n} \in W_{0}^{1,2}(\Omega): \\
-\operatorname{div}\left(M(x) \nabla u_{n}\right)+a_{n}(x) u_{n}=-\operatorname{div}\left(\frac{u_{n}}{1+\frac{1}{n}\left|u_{n}\right|} E_{n}(x)\right)+f_{n}(x),
\end{array}\right.
$$

i.e., satisfying

$$
\left[\begin{array}{l}
u_{n} \in W_{0}^{1,2}(\Omega): \int_{\Omega} M(x) \nabla u_{n} \nabla v+\int_{\Omega} a_{n}(x) u_{n} v \\
=\int_{\Omega} \frac{u_{n}}{1+\frac{1}{n}\left|u_{n}\right|} E_{n}(x) \nabla v+\int_{\Omega} f_{n}(x) v(x), \quad \forall v \in W_{0}^{1,2}(\Omega) .
\end{array}\right.
$$

Since $\psi(s)=s\left(1+\frac{s}{n}\right)^{-1}$ is increasing, we deduce from (1.7) that

$$
\left|f_{n}(x)\right|=\frac{|f(x)|}{1+\frac{1}{n}|f(x)|} \leq \frac{Q a(x)}{1+\frac{Q}{n} a(x)}=Q a_{n}(x) .
$$

We will use the following functions (introduced by De Giorgi and Stampacchia) defined for $s \in \mathbb{R}$ by

$$
G_{k}(s)=\left\{\begin{array}{ll}
0, & \text { if }|s| \leq k, \\
s-k, & \text { if } s>k, \\
s+k, & \text { if } s<-k .
\end{array} \quad T_{k}(s)= \begin{cases}s, & \text { if }|s| \leq k, \\
k, & \text { if } s>k, \\
-k, & \text { if } s<-k .\end{cases}\right.
$$

Note that a weak solution $u_{n}$ of (2.2) exists thanks to Schauder fixed point theorem. Moreover, since for every fixed $n$ the function

$$
\frac{u_{n}}{1+\frac{1}{n}\left|u_{n}\right|} \frac{E(x)}{1+\frac{R}{n}|E(x)|}
$$

belongs to $\left(L^{\infty}(\Omega)\right)^{N}$, every $u_{n}$ is bounded thanks to Stampacchia's boundedness theorem (see [23]).

Furthermore the following boundedness properties of compositions of $u_{n}$ (proved in [5]) still hold, even if in [5] there is no lower order term $a_{n}(x) u_{n}$, for the reason that, in the proofs, the positivity of $a_{n}(x)$ gives a positive term whose contribution can be dropped. 
Lemma 2.1. Assume (1.1), $f \in L^{1}(\Omega), E \in\left(L^{2}(\Omega)\right)^{N}$. Recall that $\lambda_{1}$ is the first eigenvalue of the Laplace operator. Then

$$
\lambda_{1} \int_{\Omega}\left[\log \left(1+\left|u_{n}\right|\right)\right]^{2} \leq \int_{\Omega} \frac{\left|\nabla u_{n}\right|^{2}}{\left(1+\left|u_{n}\right|\right)^{2}} \leq \frac{1}{\alpha^{2}} \int_{\Omega}|E|^{2}+\frac{2}{\alpha} \int_{\Omega}|f|
$$

which implies

$$
\text { measure }\left\{k<\left|u_{n}\right|\right\} \leq \frac{1}{[\log (1+k)]^{2}}\left[\frac{1}{\alpha^{2}} \int_{\Omega}|E|^{2}+\frac{2}{\alpha} \int_{\Omega}|f|\right] \frac{1}{\lambda_{1}}, \forall k \in \mathbb{R}^{+} .
$$

Lemma 2.2. Assume (1.1), $f \in L^{1}(\Omega), E \in\left(L^{2}(\Omega)\right)^{N}$. Then, for any $k \in \mathbb{R}^{+}$, the sequence $T_{k}\left(u_{n}\right)$ is bounded in $W_{0}^{1,2}(\Omega)$. More precisely we have:

$$
\int_{\Omega}\left|\nabla T_{k}\left(u_{n}\right)\right|^{2} \leq \frac{k^{2}}{\alpha^{2}} \int_{\Omega}|E|^{2}+k \frac{2}{\alpha} \int_{\Omega}|f|, \quad \forall k \in \mathbb{R}^{+} .
$$

Theorem 2.3. Assume (1.1), (1.2) and (1.7). Then there exists a unique weak solution $u$ of (1.5), that is a solution of (1.8). Moreover $u \in L^{\rho}(\Omega)$, for every $\rho$ and $a(x) u(x) \in L^{1}(\Omega)$.

Proof. Step 1 - The sequence $\left\{u_{n}\right\}$ is bounded in $L^{\rho}(\Omega)$, for every $\rho \geq 1$ - The use of $\frac{\left|u_{n}\right|^{2(\gamma-1)} u_{n}}{2 \gamma-1}, \gamma \geq 1$, as test function in (2.2), (2.4) and Young inequality imply that

$$
\left[\begin{array}{l}
\alpha \int_{\Omega}\left|u_{n}\right|^{2(\gamma-1)}\left|\nabla u_{n}\right|^{2}+\frac{1}{2 \gamma-1} \int_{\Omega} a_{n}(x)\left[\left|u_{n}\right|-Q\right]\left|u_{n}\right|^{2 \gamma-1} \\
\leq \int_{\Omega}\left|u_{n}\right|\left(\left|u_{n}\right|^{\gamma-1}|E|\right)\left(\left|u_{n}\right|^{\gamma-1}\left|\nabla u_{n}\right|\right) \\
\leq \frac{1}{2 \alpha} \int_{\Omega}\left|u_{n}\right|^{2}\left|u_{n}\right|^{2(\gamma-1)}|E|^{2}+\frac{\alpha}{2} \int_{\Omega}\left|u_{n}\right|^{2(\gamma-1)}\left|\nabla u_{n}\right|^{2}
\end{array}\right.
$$

which implies, thanks to the Sobolev inequality,

$$
S \frac{\alpha}{2}\left[\int_{\Omega}\left|\frac{u_{n}}{\gamma}\right|^{2^{*} \gamma}\right]^{\frac{2}{2^{*}}} \leq \frac{1}{2 \alpha} \int_{\Omega}\left|u_{n}\right|^{2 \gamma}|E|^{2}+Q^{2 \gamma} \int_{\Omega} a_{n}(x) .
$$

The inequality $\left|G_{k}\left(u_{n}\right)\right| \leq\left|u_{n}\right|$ and Hölder inequality yield

$$
\left[\begin{array}{l}
C_{\gamma}\left[\int_{\Omega}\left|G_{k}\left(u_{n}\right)\right|^{2^{*} \gamma}\right]^{\frac{2}{2^{*}}} \\
\leq \frac{2^{2 \gamma-1}}{2 \alpha} \int_{\Omega}\left|T_{k}\left(u_{n}\right)\right|^{2 \gamma}|E|^{2}+\frac{2^{2 \gamma-1}}{2 \alpha} \int_{\Omega}\left|G_{k}\left(u_{n}\right)\right|^{2 \gamma}|E|^{2}+Q^{2 \gamma} \int_{\Omega} a_{n}(x) \\
\leq \frac{2^{2 \gamma-2}}{\alpha} k^{2 \gamma} \int_{\Omega}|E|^{2}+\frac{2^{2 \gamma-2}}{\alpha}\left[\int_{\Omega}\left|G_{k}\left(u_{n}\right)\right|^{2^{*} \gamma}\right]^{\frac{2}{2^{*}}}\left[\int_{\left\{k \leq\left|u_{n}\right|\right\}}|E|^{N}\right]^{\frac{2}{N}}+Q^{2 \gamma} \int_{\Omega} a(x)
\end{array}\right.
$$

Thanks to Lemma 2.1, we can choose $k\left(\gamma,\|E\|_{N}\right)$ such that

$$
\frac{C_{\gamma}}{2} \leq \frac{2^{2 \gamma-2}}{\alpha}\left[\int_{\left\{k \leq\left|u_{n}\right|\right\}}|E|^{N}\right]^{\frac{2}{N}}, \quad k>k\left(\gamma,\|E\|_{N}\right),
$$


so that

$$
\frac{C_{\gamma}}{2}\left[\int_{\Omega}\left|G_{k}\left(u_{n}\right)\right|^{2^{*} \gamma}\right]^{\frac{2}{2^{*}}} \leq \frac{2^{2 \gamma-2}}{\alpha} k^{2 \gamma} \int_{\Omega}|E|^{2}+Q^{2 \gamma} \int_{\Omega} a(x), \quad k>k\left(\gamma,\|E\|_{N}\right) .
$$

Then, for $k>k\left(\gamma,\|E\|_{N}\right)$, from the previous inequality we deduce that

$$
\left[\begin{array}{l}
{\left[\int_{\Omega}\left|u_{n}\right|^{2^{*} \gamma}\right]^{\frac{2}{2^{*}}}=\left[\int_{\Omega}\left|T_{k}\left(u_{n}\right)+G_{k}\left(u_{n}\right)\right|^{2^{*} \gamma}\right]^{\frac{2}{2^{*}}}} \\
\leq C(k, \gamma)+C\left(Q, \gamma,\|E\|_{N}\right)\left[\int_{\Omega}|E|^{2}+\int_{\Omega} a(x)\right], \quad \forall \gamma \geq 1 .
\end{array}\right.
$$

Step 2 - The sequence $\left\{u_{n}\right\}$ is bounded in $W_{0}^{1,2}(\Omega)$ - The inequality (2.6), with $\gamma=1$ yields

$$
\frac{\alpha}{2} \int_{\Omega}\left|\nabla u_{n}\right|^{2} \leq \frac{1}{2 \alpha}\|E\|_{L^{N}}^{2}\left[\int_{\Omega}\left|u_{n}\right|^{2^{*}}\right]^{\frac{2}{2^{*}}}+Q^{2} \int_{\Omega} a(x)
$$

The conclusion follows by the boundedness proved in the previous step.

Step 3 - Passage to the limit - Thus, passing to a subsequence if necessary, we may assume the sequence $\left\{u_{n}\right\}$ converges weakly in $W_{0}^{1,2}(\Omega)$ (strongly in $L^{1}(\Omega)$ ) and weakly in $L^{\rho}(\Omega), \forall \rho$, to some $u \in W_{0}^{1,2}(\Omega) \cap L^{\rho}(\Omega)$. Moreover, if $t \in \mathbb{R}^{+}$and $\Sigma$ is a measurable subset of $\Omega$, we have

$$
\left[\begin{array}{l}
\int_{\Sigma} a_{n}(x)\left|u_{n}(x)\right| \leq t \int_{\Sigma \cap\left\{\left|u_{n}(x)\right| \leq t\right\}} a_{n}(x)+\frac{1}{t^{2 \gamma-1}} \int_{\Sigma \cap\left\{t<\left|u_{n}(x)\right|\right\}} a_{n}\left|u_{n}\right|^{2 \gamma} \\
\leq t \int_{\Sigma} a_{n}(x)+\frac{C_{1}}{t^{2 \gamma-1}},
\end{array}\right.
$$

so that (recall that the sequence $\left\{a_{n}\right\}$ is compact in $L^{1}(\Omega)$ )

$$
\lim _{\operatorname{measure}(\Sigma) \rightarrow 0} \int_{\Sigma} a_{n}(x)\left|u_{n}(x)\right| \leq 0+\frac{C_{1}}{t^{2 \gamma-1}}, \quad \forall t \in \mathbb{R}^{+},
$$

that is (equi-integrability of $a_{n}(x) u_{n}(x)$ )

$$
\lim _{\operatorname{measure}(\Sigma) \rightarrow 0} \int_{\Sigma} a_{n}(x)\left|u_{n}(x)\right|=0, \quad \text { uniformly w.r.t. } n \text {. }
$$

Since $a_{n}(x)$ and $u_{n}(x)$ converge a.e. the above equi-integrability allows us the use of the Vitali convergence theorem and we get

$$
a_{n}(x) u_{n}(x) \text { converges in } L^{1}(\Omega) \text { to } a(x) u(x) .
$$

On the other hand, the strong $L^{2}$ convergences of $u_{n}(x)$ and $E_{n}(x)$ imply that

$$
u_{n}(x) E_{n}(x) \text { converges in } L^{1}(\Omega) \text { to } u(x) E(x) .
$$

Thus it is possible to pass to the limit in (2.2) and the conclusion is done. 
Step 4 - Uniqueness - Even if the solution has finite energy, we need some carefulness, because of the singularity of the coefficients.

Here we follow $[5,6]$. We fix a $\delta \in \mathbb{R}^{+}$and in (1.8), with $f=0$ (because of the linearity of the problem), we take $\varphi=T_{h}(u), 0<h<\delta$, and we have, dropping a positive term and using the Young inequality,

$$
\alpha \int_{\Omega}\left|\nabla T_{h}(u)\right|^{2} \leq \frac{\alpha}{2} \int_{\Omega}\left|\nabla T_{h}(u)\right|^{2}+\frac{h^{2}}{2 \alpha} \int_{\{0<|u|<h\}}|E(x)|^{2},
$$

that is

$$
\frac{\alpha}{2} \int_{\Omega}\left|\nabla T_{h}(u)\right|^{2} \leq \frac{h^{2}}{2 \alpha} \int_{\{0<|u|<h\}}|E(x)|^{2}
$$

and, thanks to the Poincaré inequality,

$$
\lambda_{1} \int_{\{h<|u|\}}\left|T_{h}(u)\right|^{2} \leq \lambda_{1} \int_{\Omega}\left|T_{h}(u)\right|^{2} \leq \frac{h^{2}}{\alpha^{2}} \int_{\{0<|u|<h\}}|E(x)|^{2},
$$

which implies

$$
\lambda_{1} h^{2} \int_{\{\delta<|u|\}} 1 \leq \lambda_{1} h^{2} \int_{\{h<|u|\}} 1 \leq \frac{h^{2}}{\alpha^{2}} \int_{\{0<|u|<h\}}|E(x)|^{2} .
$$

Then, as $h \rightarrow 0$,

$$
\lambda_{1} \int_{\{\delta<|u|\}} 1 \leq 0,
$$

for every $\delta>0$. Thus $u$ is identically zero.

Example 2.4. Let $\Omega=B(0,1)=\{x:|x| \leq 1\}$. We consider the Dirichlet problem

$$
-\Delta u+a(x) u=-\operatorname{div}\left(u g(|x|) \frac{x}{|x|}\right)+f(x),
$$

with

$$
g(r)=\frac{\Lambda_{B\left(0, \frac{1}{2}\right)}}{r(-\log r)^{q}}, \frac{1}{N}<q<\frac{2}{N},
$$

where $\Lambda_{B\left(0, \frac{1}{2}\right)}(x)$ is a smooth function such that $\Lambda_{B\left(0, \frac{1}{2}\right)}(x)=1$ in $B\left(0, \frac{1}{2}\right), a(r)=\frac{2-N}{r^{2}(Q-\log r)}$ and $f(r)=Q a(r)$. We look for a radial solution $u=w(r), r=|x|$. It is possible to show that $w(r)=\log r$ is a solution with the properties stated in the previous theorem for a suitable $f(r)$. Of course $w$ is unbounded.

Theorem 2.5. Assume (1.1), (1.7) and $E \in\left(L^{\sigma}(\Omega)\right)^{N}, \sigma>N$, then the weak solution $u$ of (1.5), whose existence has been proved in Theorem 2.3, that is a solution of (1.8), belongs to $u \in W_{0}^{1,2}(\Omega) \cap L^{\infty}(\Omega)$.

Proof. Our proof follows Stampacchia's method ([23]), we take as test function in (2.2)

$$
\left(\frac{\left|u_{n}\right|}{1+\left|u_{n}\right|}-\frac{k}{1+k}\right)^{+} \frac{u_{n}}{\left|u_{n}\right|}, \quad k \geq Q
$$


and use Young inequality. Then we have

$$
\left[\begin{array}{l}
\frac{\alpha}{2} \int_{\left\{k \leq\left|u_{n}\right|\right\}} \frac{\left|\nabla u_{n}\right|^{2}}{\left(1+\left|u_{n}\right|\right)^{2}}+\int_{\Omega} a_{n}(x)\left[\left|u_{n}\right|-Q\right]\left(\frac{\left|u_{n}\right|}{1+\left|u_{n}\right|}-\frac{k}{1+k}\right)^{+} \\
\leq \frac{1}{2 \alpha} \int_{\left\{k \leq\left|u_{n}\right|\right\}}|E|^{2}
\end{array}\right.
$$

which implies, with $k=\mathrm{e}^{h}-1$ and dropping a positive term,

$$
\frac{\alpha}{2} \int_{\left\{h \leq \log \left(1+\left|u_{n}\right|\right)\right\}}\left|\nabla \log \left(1+\left|u_{n}\right|\right)\right|^{2} \leq \frac{1}{2 \alpha} \int_{\left\{h \leq \log \left(1+\left|u_{n}\right|\right)\right\}}|E|^{2} .
$$

Now, since $|E|^{2}$ belongs to $L^{r}(\Omega)$, with $r>\frac{N}{2}$, it follows from Stampacchia's theorem (see [23]) that there exists a positive constant $L$ such that

$$
\left\|\log \left(1+\left|u_{n}\right|\right)\right\|_{L^{\infty}(\Omega)} \leq L
$$

and so

$$
\left\|u_{n}\right\|_{L^{\infty}(\Omega)} \leq \mathrm{e}^{L}-1
$$

which improves Step 1 of the previous theorem. Then the conclusions about existence and uniqueness follow as in in the proof of the previous theorem, taking into account the last inequality.

Example 2.6. [Bounded solution with datum $f$ almost in $L^{1}(\Omega)$ ] Let $\Omega=B(0,1)=\{x:|x| \leq 1\}, E=A \frac{x}{|x|^{1+\theta}}$, $A \in \mathbb{R}, 0<\theta<1, a(x)=\frac{L}{|x|^{\gamma}}, L \in \mathbb{R}^{+}, 2 \theta<\gamma<N$. Note that $E \in\left(L^{\rho}(\Omega)\right)^{N}, \rho>N$. We consider the Dirichlet problem in $B(0,1)$

$$
-\Delta u+\frac{L}{|x|^{\gamma}} u=-A \operatorname{div}\left(u \frac{x}{|x|^{1+\theta}}\right)+f(x)
$$

We look for a radial solution $u=w(r), r=|x|$, so that the above Dirichlet problem turns out to be

$$
-w^{\prime \prime}(r)+w^{\prime}(r)\left[-\frac{N-1}{r}+\frac{A}{r^{\theta}}\right]+A w \frac{N-1-\theta}{r^{\theta+1}}+a(x) w=f(x)
$$

and we observe that the bounded function $w=r^{1-\theta}-1$ is a solution if

$$
\left[\begin{array}{l}
-\left\{(1-\theta)^{2}+(N-2)(1-\theta)+A(N-1-\theta)\right\} r^{-\theta-1}+A(N-2 \theta) r^{-2 \theta} \\
+L r^{1-\theta-\gamma}-L r^{-\gamma}=f(x),
\end{array}\right.
$$

where the more singular term is $r^{-\gamma}$, which has the same singularity of $a(x)$.

\subsection{Drift equation}

Here we study the impact of the interplay assumption (1.7) in the boundary value the problem (1.6) (drift problem). 
Following $[7,10,11]$, in order to study our Dirichlet problem, we introduce some approximating problems. In particular, following [11], we start by defining the following family of Dirichlet problems

$$
\left\{\begin{array}{l}
\psi_{n} \in W_{0}^{1,2}(\Omega) \\
-\operatorname{div}\left(M(x) \nabla \psi_{n}\right)+a_{n}(x) \psi_{n}=\frac{E(x) \nabla \psi_{n}}{\left(1+\frac{1}{n}|E|\right)\left(1+\frac{1}{n}\left|\nabla \psi_{n}\right|\right)}+\frac{f(x)}{1+\frac{1}{n}|f|} .
\end{array}\right.
$$

Once more, the existence of a weak solution $\psi_{n}$ is guaranteed by the Schauder theorem; moreover, since for every $n \in \mathbb{N}$ the functions on the right hand side are bounded, every $\psi_{n}$ is a bounded function (see [23]). Even if in [11] there is no lower order term $a_{n}(x) \psi_{n}$, the following properties (due to the assumptions $f \in L^{m}, m>1$, and $\left.E \in L^{N}\right)$, proved in that paper, still hold: there exists $\psi \in W_{0}^{1, m^{*}}(\Omega)$ such that

$$
\left\{\begin{array}{l}
\text { the sequence }\left\{\psi_{n}\right\} \text { converges weakly to in } W_{0}^{1, m^{*}}(\Omega) \\
\nabla \psi_{n}(x) \text { converges a.e. to } \nabla \psi(x) .
\end{array}\right.
$$

Here we prove that $\psi$ is a more regular function thanks to our interplay assumption: there exists $Q>0$ such that $|f(x)| \leq Q a(x)$.

Theorem 2.7. We assume (1.1), (1.2) and (1.7). Then there exists a unique bounded weak solution $\psi$ of (1.9).

Proof. We use $G_{k}\left(\psi_{n}\right)$ as test function in the weak formulation of the Dirichlet problem (2.9)

$$
\left\{\begin{array}{l}
u_{n} \in W_{0}^{1,2}(\Omega): \\
\int_{\Omega} M(x) \nabla \psi_{n} \nabla v+\int_{\Omega} a_{n}(x) \psi_{n} v \\
=\int_{\Omega} \frac{E(x) \nabla \psi_{n}}{\left(1+\frac{1}{n}|E|\right)\left(1+\frac{1}{n}\left|\nabla \psi_{n}\right|\right)} v+\int_{\Omega} \frac{f(x)}{1+\frac{1}{n}|f|} v \\
\forall v \in W_{0}^{1,2}(\Omega)
\end{array}\right.
$$

and we obtain

$$
\left[\begin{array}{l}
\alpha \int_{\Omega}\left|\nabla G_{k}\left(\psi_{n}\right)\right|^{2}+\int_{\Omega} a_{n}(x)\left|\psi_{n}\right|\left|G_{k}\left(\psi_{n}\right)\right| \\
\leq \int_{\Omega}|E(x)|\left|\nabla G_{k}\left(\psi_{n}\right)\right|\left|G_{k}\left(\psi_{n}\right)\right|+Q \int_{\Omega} a_{n}(x)\left|G_{k}\left(\psi_{n}\right)\right| .
\end{array}\right.
$$

Then

$$
\alpha \int_{\Omega}\left|\nabla G_{k}\left(\psi_{n}\right)\right|^{2}+\int_{\Omega} a_{n}(x)\left[\left|\psi_{n}\right|-Q\right]\left|G_{k}\left(\psi_{n}\right)\right| \leq \int_{\Omega}|E(x)|\left|\nabla G_{k}\left(\psi_{n}\right)\right|\left|G_{k}\left(\psi_{n}\right)\right|
$$

If $k \geq Q$, we drop the second (positive) integral and we have

$$
\alpha \int_{\Omega}\left|\nabla G_{k}\left(\psi_{n}\right)\right|^{2} \leq\left[\int_{\left\{k<\left|\psi_{n}\right|\right\}}|E|^{N}\right]^{\frac{2}{N}} \mathcal{S} \int_{\Omega}\left|\nabla G_{k}\left(\psi_{n}\right)\right|^{2},
$$


where we have used Hölder and Sobolev inequalities. If $\int_{\Omega}\left|\nabla G_{k}\left(\psi_{n}\right)\right|^{2}>0$, for $k \geq Q$, then we have

$$
\frac{\alpha}{\mathcal{S}} \leq\left[\int_{\left\{k<\left|\psi_{n}\right|\right\}}|E|^{N}\right]^{\frac{2}{N}}, \quad k \geq Q .
$$

From (2.10) we deduce the boundedness of $\left\{\psi_{n}\right\}$ in $L^{1}(\Omega)$. Thus there exists a constant $C(E, f)>0$ such that

$$
k \int_{\left\{k<\left|\psi_{n}\right|\right\}} 1 \leq \int_{\left\{k<\left|\psi_{n}\right|\right\}}\left|\psi_{n}\right| \leq \int_{\Omega}\left|\psi_{n}\right| \leq C(E, f)
$$

which implies

$$
\text { measure }\left\{k<\left|\psi_{n}\right|\right\} \leq \frac{C(E, f)}{k},
$$

that is, the measure of the set $\left\{k<\left|\psi_{n}\right|\right\}$, for $k$ large is small, uniformly with respect to $n$. Thus the absolute continuity of the Lebesgue integral yields the existence of $k^{*} \geq Q$ such that

$$
\left[\int_{\left\{k^{*}<\left|\psi_{n}\right|\right\}}|E|^{N}\right]^{\frac{2}{N}} \leq \frac{\alpha}{2 \mathcal{S}}
$$

in contradiction with (2.12). Then there exists $\tilde{M} \geq Q$ such that $\left|\psi_{n}\right| \leq \tilde{M}$.

Now we use $\psi_{n}$ as test function in the weak formulation of the Dirichlet problem (2.9) and we have, dropping a positive term

$$
\alpha \int_{\Omega}\left|\nabla \psi_{n}\right|^{2} \leq \tilde{M}\left[\int_{\Omega}|E|^{2}\right]^{\frac{1}{2}}\left[\int_{\Omega}\left|\nabla \psi_{n}\right|^{2}\right]^{\frac{1}{2}}+\tilde{M} \int_{\Omega}|f|,
$$

which implies the boundedness in $W_{0}^{1,2}(\Omega)$ of the sequence $\left\{\psi_{n}\right\}$. Thus, passing to a subsequence if necessary, we may assume the sequence $\left\{\psi_{n}\right\}$ converges weakly in $W_{0}^{1,2}(\Omega)$ and *-weakly in $L^{\infty}(\Omega)$ and a.e. to some $\psi \in W_{0}^{1,2}(\Omega) \cap L^{\infty}(\Omega)$.

In order to pass to the limit, we need to study the convergence of $\frac{E(x) \nabla \psi_{n}}{\left(1+\frac{1}{n}|E|\right)\left(1+\frac{1}{n}\left|\nabla \psi_{n}\right|\right)}$. The sequence is bounded in $L^{\frac{2 N}{N+2}}$ and converges a.e. (recall (2.10)) so that it converges strongly in $L^{\sigma}, 1 \leq \sigma<\frac{2 N}{N+2}$. On the other hand, $a_{n}(x) \psi_{n}$ converges in $L^{1}$ since it is dominated by the convergent sequence $\left\{\tilde{M} a_{n}(x)\right\}$.

Now it is easy to pass to the limit in (2.11) and to prove that $\psi$ is a bounded weak solution $\psi$ of (1.9).

Remark 2.8. We point out the differences between the summability assumptions on $E$ in the Theorems 2.5 and 2.7 .

\section{THE IMPACT OF INTERPLAY BETWEEN THE ZERO ORDER TERM AND THE TERMS OF ORDER ONE}

The main assumption of this section is the interplay inequality (1.10), with or without restrictions on the size of the constant $R$. 


\subsection{Convection equation}

Remark 3.1. Consider the boundary value problem

$$
\left\{\begin{aligned}
-\operatorname{div}(M(x) \nabla u)+\mu \frac{u}{|x|^{2}}=-A \operatorname{div}\left(u \frac{x}{|x|^{2}}\right)+f(x), & \text { in } \Omega \\
u=0, & \text { on } \partial \Omega
\end{aligned}\right.
$$

where $0 \in \Omega, \mu \in \mathbb{R}^{+}, A \in \mathbb{R}$. The inequality (1.10) (where $a(x)=\mu \frac{u}{|x|^{2}}$ and $E(x)=A \frac{x}{|x|^{2}}$ ) is satisfied by the real numbers $R>0$ such that $\frac{A^{2}}{\mu} \leq R$. In [13], the existence of very singular distributional solutions $u \in W_{0}^{1, q}(\Omega)$, for every $q<\frac{N \alpha}{|A|+\alpha}<\frac{N}{N-1}$ is proved if $\alpha(N-2) \leq|A|<\alpha(N-1), f \in L^{1}(\Omega)$.

Thanks to the following theorems, this existence result can be improved: solutions with more summability, no restrictions on the size of $|A|$.

In this first theorem under the assumption (1.10), we prove the existence of distributional solutions without restrictions on the size of $R$; conversely, in Theorem 3.5, we need a restriction on $R$.

Theorem 3.2. Assume (1.1), (1.7), (1.10) and

$$
|f| \log (1+|f|) \in L^{1}(\Omega)
$$

Then there exists a distributional solution $u \in W_{0}^{1, \frac{N}{N-1}}(\Omega)$ of $(1.5)$, that is a solution of

$$
\int_{\Omega} M(x) \nabla u \nabla \varphi+\int_{\Omega} a(x) u \varphi=\int_{\Omega} u E(x) \nabla \varphi+\int_{\Omega} f(x) \varphi(x) .
$$

Moreover $a(x) u$ and $u E(x)$ belong to $L^{1}$.

Proof. Step 1 - A priori estimates - Use $g\left(u_{n}\right)$ as test function in (2.3), where $g(t)=\log (1+|t|) \frac{t}{|t|}$. Then we have

$$
\left[\begin{array}{l}
\frac{\alpha}{2} \int_{\Omega}\left|\nabla u_{n}\right|^{2} g^{\prime}\left(u_{n}\right)+\int_{\Omega} a_{n}(x)\left|u_{n}\right|\left|g\left(u_{n}\right)\right| \\
\leq \frac{1}{2 \alpha} \int_{\Omega} u_{n}^{2} g^{\prime}\left(u_{n}\right)\left|E_{n}\right|^{2}+\int_{\Omega}\left|f_{n}(x)\right|\left|g\left(u_{n}\right)\right| .
\end{array}\right.
$$

The interplay assumption yields

$$
\frac{\alpha}{2} \int_{\Omega}\left|\nabla u_{n}\right|^{2} g^{\prime}\left(u_{n}\right)+\int_{\Omega} a_{n}(x)\left\{\left|u_{n}\right|\left|g\left(u_{n}\right)\right|-\frac{R}{2 \alpha} u_{n}^{2} g^{\prime}\left(u_{n}\right)\right\} \leq \int_{\Omega}|f|\left|g\left(u_{n}\right)\right|,
$$

that is

$$
\left[\begin{array}{l}
\frac{\alpha}{2} \int_{\Omega} \frac{\left|\nabla u_{n}\right|^{2}}{1+\left|u_{n}\right|}+\int_{\Omega} a_{n}(x)\left|u_{n}\right| \log \left(1+\left|u_{n}\right|\right) \\
\leq \int_{\Omega} a(x) \frac{R}{2 \alpha} \frac{u_{n}^{2}}{1+\left|u_{n}\right|}+\int_{\Omega}|f| \log \left(1+\left|u_{n}\right|\right)
\end{array}\right.
$$

Here we use the inequality

$$
s t \leq s \log (s)-s+e^{t}-1, \quad s, t \in \mathbb{R}^{+},
$$


which implies

$$
s \log (1+r) \leq s \log (s)-s+r \leq s \log (1+s)+r
$$

Thus we proved

$$
\left[\begin{array}{l}
\frac{\alpha}{2} \int_{\Omega} \frac{\left|\nabla u_{n}\right|^{2}}{1+\left|u_{n}\right|}+\int_{\Omega} a_{n}(x)\left|u_{n}\right|\left[\log \left(1+\left|u_{n}\right|\right)-\frac{R}{2 \alpha}\right] \\
\leq \int_{\Omega}|f| \log (1+|f|)+\int_{\Omega}\left|u_{n}\right|,
\end{array}\right.
$$

which implies

$$
\frac{\alpha}{2} \int_{\Omega} \frac{\left|\nabla u_{n}\right|^{2}}{1+\left|u_{n}\right|} \leq \int_{\Omega}|f| \log (1+|f|)+\int_{\Omega}\left|u_{n}\right|+\frac{R}{2 \alpha}\left(e^{\frac{R}{2 \alpha}}-1\right) \int_{\Omega} a_{n}(x)
$$

The above inequality implies that the sequence $\left\{\sqrt{\left|u_{n}\right|}\right\}$ is bounded in $W_{0}^{1,2}(\Omega)$ and then (see also [12]) the sequence $\left\{u_{n}\right\}$ is bounded in $W_{0}^{1, \frac{N}{N-1}}(\Omega)$. Moreover

$$
\text { the sequence } \int_{\Omega} a_{n}(x)\left|u_{n}\right| \log \left(1+\left|u_{n}\right|\right) \text { is bounded in } L^{1}(\Omega) \text {. }
$$

Thus, passing to a subsequence if necessary, we may assume the sequence $\left\{u_{n}\right\}$ converges weakly in $W_{0}^{1, \frac{N}{N-1}}(\Omega)$ and a.e. to some $u \in W_{0}^{1, \frac{N}{N-1}}(\Omega)$.

Step 2 - Existence - Since $a_{n}(x)$ and $u_{n}(x)$ converge a.e. in order to prove the $L^{1}$ convergence of $a_{n}(x) u_{n}(x)$ with Vitali theorem, we need to prove the equi-integrability of the sequence. Indeed, for every measurable subset $\Sigma \subset \Omega$ we have ( $t$ arbitrary real positive number)

$$
\left[\begin{array}{l}
\int_{\Sigma} a_{n}(x)\left|u_{n}(x)\right| \\
\leq t \int_{\Sigma} a_{n}(x)+\frac{1}{\log (1+t)} \int_{\Sigma \cap\left\{t<\left|u_{n}(x)\right|\right\}} a_{n}(x)\left|u_{n}\right| \log \left(1+\left|u_{n}\right|\right),
\end{array}\right.
$$

which implies, $\forall t \in \mathbb{R}^{+}$, since the sequence $\left\{u_{n}\right\}$ is compact in $L^{1}$,

$$
\lim _{\text {measure }(\Sigma) \rightarrow 0} \int_{\Sigma} a_{n}(x)\left|u_{n}(x)\right| \leq \frac{C_{1}}{\log (1+t)} \text {, uniformly with respect to } n
$$

that is

$$
\lim _{\text {measure }(\Sigma) \rightarrow 0} \int_{\Sigma} a_{n}(x)\left|u_{n}(x)\right|=0, \quad \text { uniformly with respect to } n \text {. }
$$

Thus we proved the $L^{1}$ convergence of $a_{n}(x) u_{n}(x)$.

On the other hand, the assumption (1.10) implies that also the sequence $\left\{\left|E_{n}(x)\right|^{2} u_{n}(x)\right\}$ is bounded in $L^{1}(\Omega)$. Now we prove the strong $L^{1}$ convergence of the sequence $\left\{E_{n}(x) u_{n}(x)\right\}$. Since $E_{n}(x)$ and $u_{n}(x)$ converge a.e. in order to prove the convergence with Vitali theorem, we need to prove the equi-integrability of the sequence. 
Indeed, for every measurable subset $\Sigma \subset \Omega$ we have ( $t$ arbitrary real positive number)

$$
\left[\begin{array}{l}
\int_{\Sigma}\left|E_{n}(x)\right|\left|u_{n}(x)\right| \leq t \int_{\Sigma \cap\left\{\left|E_{n}(x)\right| \leq t\right\}}\left|u_{n}\right|+\int_{\Sigma \cap\left\{t<\left|E_{n}(x)\right|\right\}}\left|E_{n}\right|\left|u_{n}\right| \\
\leq t \int_{\Sigma}\left|u_{n}\right|+\frac{1}{t} \int_{\Omega}\left|E_{n}\right|^{2}\left|u_{n}\right| \leq t \int_{\Sigma}\left|u_{n}\right|+\frac{1}{t} C_{1},
\end{array}\right.
$$

which implies, $\forall t \in \mathbb{R}^{+}$, since the sequence $\left\{u_{n}\right\}$ is compact in $L^{1}$,

$$
\lim _{\operatorname{measure}(\Sigma) \rightarrow 0} \int_{\Sigma}\left|E_{n}(x)\right|\left|u_{n}(x)\right| \leq \frac{C_{1}}{t} \text {, uniformly with respect to } n
$$

that is

$$
\lim _{\text {measure }(\Sigma) \rightarrow 0} \int_{\Sigma}\left|E_{n}(x)\right|\left|u_{n}(x)\right|=0, \quad \text { uniformly with respect to } n,
$$

At this time, it is possible to pass to the limit in (2.3) (for every smooth function $v$ ) and it is proved that $u$ is a distributional solution of the Dirichlet problem (3.3).

Remark 3.3. We point out that that, even if $f \in L^{1}(\Omega)$ and $E \in\left(L^{2}(\Omega)\right)^{N}$ are very singular, in the above theorem is proved the existence of a distributional solution belonging to the Sobolev space $W_{0}^{1, q}(\Omega)$, thanks to the interplay assumption (1.10). Without this assumption, it is needed a very weak definition ("entropy solution" introduced in [4]) of solution whose existence, outside of $W_{0}^{1,1}(\Omega)$, is proved in [6].

Remark 3.4. About the uniqueness, we recall the Serrin's counterexample, in [22], about the non uniqueness of infinite energy solutions, even in the linear case, i.e. $E=0$.

In the previous theorem, the existence of infinite energy (i.e. distributional) solutions is proved without restrictions on the size of $R$; in the next theorem we prove the existence of finite energy (i.e. weak) solutions with the restriction $R<4 \alpha$.

Theorem 3.5. Assume (1.1), (1.10), with the constraint

$$
R<4 \alpha
$$

and

$$
f \in L^{m}(\Omega), m \geq \frac{2 N}{N+2} .
$$

Then there exists a unique weak solution $u \in W_{0}^{1,2}(\Omega)$ of (1.5), that is a solution of (3.3), for every $\varphi \in$ $W_{0}^{1,2}(\Omega) \cap L^{\infty}(\Omega)$. Moreover $a(x) u$ and $u E(x)$ belong to $L^{2}$. 
Proof. We use $u_{n}$ as test function in (2.3), the Young inequality and we have, with $0<B<4 \alpha$,

$$
\left[\begin{array}{l}
\alpha \int_{\Omega}\left|\nabla u_{n}\right|^{2}+\int_{\Omega} a_{n}(x)\left|u_{n}\right|^{2} \leq \int_{\Omega}\left|u_{n}\right|\left|E_{n}\right|\left|\nabla u_{n}\right|+\int_{\Omega}\left|f_{n}(x)\right|\left|u_{n}\right| \\
\leq B \int_{\Omega}\left|\nabla u_{n}\right|^{2}+\frac{1}{4 B} \int_{\Omega}\left|E_{n}\right|^{2}\left|u_{n}\right|^{2}+\int_{\Omega}\left|f_{n}(x)\right|\left|u_{n}\right| \\
\leq B \int_{\Omega}\left|\nabla u_{n}\right|^{2}+\frac{R}{4 B} \int_{\Omega} a_{n}(x)\left|u_{n}\right|^{2}+\int_{\Omega}|f(x)|\left|u_{n}\right| .
\end{array}\right.
$$

Thus

$$
\epsilon \int_{\Omega}\left|\nabla u_{n}\right|^{2}+\left(1-\frac{R}{4 B}\right) \int_{\Omega} a_{n}(x)\left|u_{n}\right|^{2} \leq \int_{\Omega}|f(x)|\left|u_{n}\right|
$$

which implies, thanks to (3.6), for some positive constants $C_{i}$,

$$
\left\{\begin{array}{l}
{\left[\int_{\Omega}\left|\nabla u_{n}\right|^{2}\right]^{\frac{1}{2}} \leq C_{1}\|f\|_{L^{\frac{2 N}{N+2}}(\Omega)}} \\
\int_{\Omega} a_{n}(x)\left|u_{n}\right|^{2} \leq C_{2}
\end{array}\right.
$$

and, thanks to (1.10),

$$
\frac{1}{R} \int_{\Omega}\left|E_{n}\right|^{2}\left|u_{n}\right|^{2} \leq C_{2}
$$

that is, the sequence $\left\{\left|E_{n} \| u_{n}\right|\right\}$ is bounded in $L^{2}$.

We conclude that the sequence $\left\{u_{n}\right\}$ is bounded in $W_{0}^{1,2}(\Omega)$ and (passing to a subsequence if necessary) convergent weakly in $W_{0}^{1,2}(\Omega)$, strongly in $L^{s}(\Omega), 1 \leq s<2^{*}$, and a.e. to some $u$. Moreover $u_{n} E_{n}$ converges weakly in $L^{2}$, since it converges a.e. and it is bounded in $L^{2}$.

Furthermore $a_{n} u_{n}$ converges in $L^{1}(\Omega)$, once more thanks to the Vitali theorem, since we have the a.e. convergence and the equi-integrability that we sketch below. Indeed, for every measurable subset $\Sigma \subset \Omega$ we have ( $t$ arbitrary real positive number)

$$
\int_{\Sigma} a_{n}(x)\left|u_{n}(x)\right| \leq t \int_{\Sigma} a_{n}(x)+\frac{C_{2}}{t} .
$$

Then it is straightforward to conclude that $u$ is a weak solution of (3.3); moreover the uniqueness can be proved exactly as in Theorem 2.3.

In order to better understand the differences on the assumptions on $R$ in the previous theorems, below, we sketch the proof of the boundedness of the sequence $\left\{u_{n}\right\}$, if we assume $f \in L^{m}(\Omega), 1<m<\frac{N}{2}$, and (1.10). We will see that our proof needs $R<4 \alpha \frac{m^{\prime}}{m^{* *}}$. Thus $m=\frac{2 N}{N+2}$ gives $R<4 \alpha$ (last theorem) and $m \rightarrow 1$ gives no restrictions on $R$ (first theorem).

Proposition 3.6. Assume (1.1) and

$$
|E|^{2} \leq R a(x) \in L^{1}(\Omega), \quad R<4 \alpha \frac{m^{\prime}}{m^{* *}} .
$$


$f \in L^{m}(\Omega)$ with $m>1$. Then the sequence $\left\{u_{n}\right\}$ is bounded in $L^{m^{* *}}(\Omega)$.

Sketch of the proof. We follow [12]. Let $\gamma=\frac{m^{* *}}{2^{*}}$, so that $\gamma>\frac{1}{2}$. Note that $2^{*} \gamma=m^{* *}$ and that $m^{\prime}(2 \gamma-1)=$ $m^{* *}$. Use in $(2.3) g\left(u_{n}\right)=\left[\left(h+\left|u_{n}\right|\right)^{2 \gamma-1}-h^{2 \gamma^{-1}}\right] \frac{u_{n}}{\left|u_{n}\right|}, h \in \mathbb{R}^{+}$, as test function. Once more, we use the Young inequality; then the limit as $h \rightarrow 0$ yields

$$
\left[\begin{array}{l}
C_{\gamma}\left[\int_{\Omega}\left|u_{n}\right|^{\gamma^{*}}\right]^{\frac{2}{2^{*}}}+\left[1-\frac{R}{4 B}(2 \gamma-1)\right] \int_{\Omega} a_{n}(x)\left|u_{n}\right|^{2 \gamma} \\
\leq(\alpha-B)(2 \gamma-1) \int_{\Omega}\left|\nabla u_{n}\right|^{2}\left|u_{n}\right|^{2 \gamma-2}+\left[1-\frac{R}{4 B}(2 \gamma-1)\right] \int_{\Omega} a_{n}(x)\left|u_{n}\right|^{2 \gamma} \\
\leq \int_{\Omega}\left|f_{n}(x)\right|\left|u_{n}\right|^{2 \gamma-1}
\end{array}\right.
$$

We need $\frac{R}{4 B}(2 \gamma-1)<1$, that is $\frac{R}{4}\left(2 \frac{m^{* *}}{2^{*}}-1\right)<B<\alpha$. Recall that $B<\alpha$, so that we have the condition $\frac{R}{4}\left(2 \frac{m^{* *}}{2^{*}}-1\right)<\alpha$, which is equivalent to (3.10). Then the inequality (3.11) implies that the sequence $\left\{u_{n}\right\}$ is bounded in $L^{m^{* *}}(\Omega)$.

\subsubsection{Double interplay}

In this subsection we assume both (1.7) and (1.10) and we slightly improve the existence result of Theorem 3.5.

Theorem 3.7. Assume (1.1), (1.7), (1.10). Then there exists a distributional solution $u \in W_{0}^{1, \frac{N}{N-1}}(\Omega)$ of $(1.5)$, that is a solution of (3.3). Moreover $a(x) u$ and $u E(x)$ belong to $L^{1}$.

Proof. Step 1 - A priori estimates - Use $g\left(u_{n}\right)$ as test function in (2.3), where $g(t)=\log (1+|t|) \frac{t}{|t|}$. Then we have

$$
\frac{\alpha}{2} \int_{\Omega}\left|\nabla u_{n}\right|^{2} g^{\prime}\left(u_{n}\right)+\int_{\Omega} a_{n}(x)\left[u_{n}-f_{n}(x)\right] g\left(u_{n}\right) \leq \frac{1}{2 \alpha} \int_{\Omega} u_{n}^{2} g^{\prime}\left(u_{n}\right)\left|E_{n}\right|^{2} .
$$

The two interplay assumptions yield

$$
\frac{\alpha}{2} \int_{\Omega}\left|\nabla u_{n}\right|^{2} g^{\prime}\left(u_{n}\right)+\int_{\Omega} a_{n}(x)\left\{\left[\left|u_{n}\right|-Q\right]\left|g\left(u_{n}\right)\right|\right\} \leq \int_{\Omega} \frac{R}{2 \alpha} a_{n}(x) u_{n}^{2} g^{\prime}\left(u_{n}\right),
$$

that is

$$
\frac{\alpha}{2} \int_{\Omega} \frac{\left|\nabla u_{n}\right|^{2}}{1+\left|u_{n}\right|}+\int_{\Omega} a_{n}(x)\left\{\left[\left|u_{n}\right|-Q\right] \log \left(1+\left|u_{n}\right|\right)\right\} \leq \int_{\Omega} a_{n}(x) \frac{R}{2 \alpha} \frac{u_{n}^{2}}{1+\left|u_{n}\right|}
$$

which implies

$$
\frac{\alpha}{2} \int_{\Omega} \frac{\left|\nabla u_{n}\right|^{2}}{1+\left|u_{n}\right|}+\int_{\Omega} a_{n}(x)\left\{\left[\left|u_{n}\right| \log \left(1+\left|u_{n}\right|\right)-Q \log \left(1+\left|u_{n}\right|\right)-\frac{R}{2 \alpha}\left|u_{n}\right|\right\} \leq 0 .\right.
$$


Note that, if

$$
\left\{\begin{array}{l}
\left|u_{n}\right| \geq Q \\
\left|u_{n}\right| \geq e^{\frac{R}{2 \alpha}}-1
\end{array}\right.
$$

then

$$
\left|u_{n}\right| \log \left(1+\left|u_{n}\right|\right)-Q \log \left(1+\left|u_{n}\right|\right)-\frac{R}{2 \alpha}\left|u_{n}\right| \geq 0
$$

so that, if we define $T=\max \left(Q, e^{\frac{R}{2 \alpha}}-1\right)$, we have

$$
\left[\begin{array}{l}
\frac{\alpha}{2} \int_{\Omega} \frac{\left|\nabla u_{n}\right|^{2}}{1+\left|u_{n}\right|} \leq \int_{\left\{\left|u_{n}\right|<T\right\}} a_{n}(x)\left[Q \log \left(1+\left|u_{n}\right|\right)+\frac{R}{2 \alpha}\left|u_{n}\right|\right] \\
\leq\left[Q \log (1+T)+\frac{R}{2 \alpha} T\right] \int_{\Omega} a(x)
\end{array}\right.
$$

The above inequality implies that the sequence $\left\{\sqrt{\left|u_{n}\right|}\right\}$ is bounded in $W_{0}^{1,2}(\Omega)$ and then (see also [12]) the sequence $\left\{u_{n}\right\}$ is bounded in $W_{0}^{1, \frac{N}{N-1}}(\Omega)$.

Step 2 - Existence - It is the same of the proof of Theorem 3.5.

Example 3.8. $\left[|E|^{2}=4 \alpha a(x)\right.$ : instead of (3.6)]

Let $\Omega=B(0,1)=\{x:|x| \leq 1\}$. We consider the Dirichlet problem

$$
-\Delta u+\frac{A^{2}}{4|x|^{2}} u=-A \operatorname{div}\left(u \frac{x}{|x|^{2}}\right)+f(x) .
$$

We look for $w=\left[r^{q}-1\right], q>2-N$. Thus we choose $q=\frac{2-N}{2}, A=2-N$ and the above Dirichlet problem becomes

$$
\frac{3(N-2)^{2}}{4 r^{2}}=f(x)
$$

Summarizing we have

$$
\left\{\begin{array}{l}
|E|^{2}=4 \alpha a(x) \\
f \in L^{\rho}(\Omega), \rho<\frac{N}{2} \\
\text { but } u \notin W_{0}^{1,2}(\Omega): u \in M^{2^{*}}(\Omega) .
\end{array}\right.
$$

\subsection{Drift equation}

Theorem 3.9. We assume (1.1) and (1.10) with the constraint (3.6) and

$$
f \in L^{m}(\Omega), m \geq \frac{2 N}{N+2} .
$$

Then there exists a weak solution $\psi \in W_{0}^{1,2}(\Omega)$ of (1.6), that is a solution of (1.9), for every $\varphi \in W_{0}^{1,2}(\Omega)$. Moreover $a(x) \psi$ and $\psi E(x)$ belong to $L^{2}$. 
Proof. Step 1 - Estimates - We use $\psi_{n}$ as test function in (2.9) and we have (B such that $\left.\frac{R}{4}<B<\alpha\right)$

$$
\left[\begin{array}{l}
(\alpha-B) \int_{\Omega}\left|\nabla \psi_{n}\right|^{2}+\int_{\Omega} a_{n}(x)\left|\psi_{n}\right|^{2} \\
\leq \frac{R}{4 B} \int_{\Omega} a_{n}(x)\left|\psi_{n}\right|^{2}+\int_{\Omega}\left|f_{n}(x)\right|\left|\psi_{n}\right|
\end{array}\right.
$$

which implies

$$
(\alpha-B) \int_{\Omega}\left|\nabla \psi_{n}\right|^{2}+\left(1-\frac{R}{4 B}\right) \int_{\Omega} a_{n}(x)\left|\psi_{n}\right|^{2} \leq \int_{\Omega}\left|f_{n}(x)\right|\left|\psi_{n}\right|
$$

Then, thanks our assumptions, we drop a positive term and we have

$$
(\alpha-B) \int_{\Omega}\left|\nabla \psi_{n}\right|^{2} \leq \int_{\Omega}|f(x)|\left|\psi_{n}\right| \leq\|f\|_{L^{\frac{2 N}{N+2}}(\Omega)} \frac{1}{\mathcal{S}}\left[\int_{\Omega}\left|\nabla \psi_{n}\right|^{2}\right]^{\frac{1}{2}}
$$

Thus we proved

$$
(\alpha-B)\left[\int_{\Omega}\left|\nabla \psi_{n}\right|^{2}\right]^{\frac{1}{2}} \leq\|f\|_{L^{\frac{2 N}{N+2}}(\Omega)} \frac{1}{\mathcal{S}}
$$

We conclude that the sequence $\left\{\psi_{n}\right\}$ is bounded in $W_{0}^{1,2}(\Omega)$ and (passing to a subsequence if necessary) convergent weakly in $W_{0}^{1,2}(\Omega)$, strongly in $L^{s}(\Omega), 1 \leq s<2^{*}$, and a.e. to some $\psi$.

Step 2 - Limits - The proof of the convergence of $a_{n} \psi_{n}$ in $L^{1}$ is the same of the convergence of $a_{n} u_{n}$ in $L^{1}$, see the proof of Theorem 3.5.

As a consequence of the last inequality used in (3.12), the sequence $\int_{\Omega} a_{n}(x)\left|\psi_{n}\right|^{2}$ is bounded and then (because of the assumption (1.10)) the sequence $\left\{\psi_{n} E_{n}\right\}$ is bounded in $L^{2}$. Moreover $\psi_{n} E_{n}$ converges weakly in $L^{2}$ to $\psi E$, since it converges a.e. and it is bounded in $L^{2}$, which ends the proof.

In order to better understand the role played by the size of $R$, as in Proposition 3.6, it is possible to prove the following result.

Proposition 3.10. The sequence $\left\{\psi_{n}\right\}$ is bounded in $L^{m^{* *}}(\Omega)$, if we assume $f \in L^{m}(\Omega), 1<m<\frac{N}{2}$, $(1.10)$ and $R<4 \alpha \frac{m^{* *}}{m^{\prime}}$.

Remark 3.11. Note that the last inequality, concerning the drift problem, contains the restriction $\frac{R}{4 \alpha}<\frac{m^{* *}}{m^{\prime}}$, while the inequality $(3.10)$, concerning the convection problem, contains the reciprocal restriction $\frac{R}{4 \alpha}<\frac{m^{\prime}}{m^{* *}}$.

\subsubsection{Double interplay}

In this subsection we assume both (1.7) and (1.10) and we improve the existence result of Theorem 3.9 .

Theorem 3.12. We assume (1.1), (1.7) and (1.10) with the constraint (3.6) and

$$
f \in L^{1}(\Omega)
$$

Then there exists a bounded weak solution $\psi \in W_{0}^{1,2}(\Omega)$ of (1.6), that is a solution of (1.9), for every $\varphi \in$ $W_{0}^{1,2}(\Omega)$. Moreover $a(x) \psi$ and $\psi E(x)$ belong to $L^{2}$. 
Proof. Step 1 - Estimates - We use $G_{k}\left(\psi_{n}\right), k \in \mathbb{R}^{+}$, as test function in (2.9) and we have (B such that $\left.\frac{R}{4}<B<\alpha\right)$, thanks to the Young inequality and to the interplay assumptions,

$$
(\alpha-B) \int_{\Omega}\left|\nabla G_{k}\left(\psi_{n}\right)\right|^{2}+\int_{\Omega} a_{n}(x)\left(\left|\psi_{n}\right|-\frac{R}{4 B}\left|G_{k}\left(\psi_{n}\right)\right|-Q\right)\left|G_{k}\left(\psi_{n}\right)\right| \leq 0,
$$

Thus if $k \geq Q$, we note that $\left|\psi_{n}\right|>Q$ implies $\left|\psi_{n}\right|=Q+\left|G_{Q}\left(\psi_{n}\right)\right|$; so that

$$
\left|\psi_{n}\right|-\frac{R}{4 B}\left|G_{Q}\left(\psi_{n}\right)\right|-Q=\left(1-\frac{R}{4 B}\right)\left|G_{Q}\left(\psi_{n}\right)\right| \geq 0
$$

which implies in (3.13) that

$$
\left|\psi_{n}\right| \leq Q
$$

Moreover the use of $\psi_{n}$, as test function in (2.9) yields

$$
(\alpha-B) \int_{\Omega}\left|\nabla \psi_{n}\right|^{2} \leq Q^{2}\left(\frac{R}{4 B}+1\right) \int_{\Omega} a(x)
$$

Step 2 - Existence - It is the same of the proof of Theorem 3.9.

Acknowledgements. The author acknowledges the support of Eu.gi.27920.

\section{REFERENCES}

[1] D. Arcoya and L. Boccardo, Regularizing effect of the interplay between coefficients in some elliptic equations. J. Funct. Anal. 268 (2015) 1153-1166.

[2] D. Arcoya and L. Boccardo, Regularizing effect of $L^{q}$ interplay between coefficients in some elliptic equations. J. Math. Pures Appl. 111 (2018) 106-125.

[3] D. Arcoya, L. Boccardo and L. Orsina, Regularizing effect of the interplay between coefficients in some nonlinear Dirichlet problems with distributional data. Ann. Mat. Pura Appl. 199 (2020) 1909-1921.

[4] P. Bénilan, L. Boccardo, T. Gallouët, R. Gariepy, M. Pierre and J.L. Vázquez, An $L^{1}$-theory of existence and uniqueness of solutions of nonlinear elliptic equations. Ann. Scuola Norm. Sup. Pisa Cl. Sci. 22 (1995) 241-273.

[5] L. Boccardo, Some developments on Dirichlet problems with discontinuous coefficients. Boll. U.M.I. 2 (2009) $285-297$.

[6] L. Boccardo, Dirichlet problems with singular convection terms and applications. J. Differ. Equ. 258 (2015) 22902314.

[7] L. Boccardo, Stampacchia-Calderon-Zygmund theory for linear elliptic equations with discontinuous coefficients and singular drift. ESAIM: COCV 25 (2019) 47.

[8] L. Boccardo, Finite energy weak solutions to some Dirichlet problems with very singular drift. Differ. Integr. Equ. 32 (2019) 409-422.

[9] L. Boccardo and S. Buccheri, A nonlinear homotopy between two linear Dirichlet problems. Rev. Mat. Complut. 34 (2021) $541-558$.

[10] L. Boccardo, S. Buccheri and G.R. Cirmi, Two linear noncoercive dirichlet problems in duality. Milan J. Math. 86 (2018) 97-104.

[11] L. Boccardo, S. Buccheri and G.R. Cirmi, Calderon-Zygmund-Stampacchia theory for infinite energy solutions of nonlinear elliptic equations with singular drift. NoDEA Nonlinear Differ. Equ. Appl. 27 (2020) 38.

[12] L. Boccardo and T. Gallouët, Nonlinear elliptic equations with right hand side measures. Commun. Partial Differ. Equ. 17 (1992) 641-655.

[13] L. Boccardo and L. Orsina, Very singular solutions for linear Dirichlet problems with singular convection terms. To appear Nonlinear Anal. TMA.

[14] G. Bottaro and M.E. Marina, Problema di Dirichlet per equazioni ellittiche di tipo variazionale su insiemi non limitati. Boll. U. M. I. 8 (1973) 46-56.

[15] S. Buccheri, Gradient estimates for nonlinear elliptic equations with first order terms. Manuscr. Math. (2020) https://doi.org/ 10.1007/s00229-020-01210-5.

[16] S. Buccheri, The Bottaro-Marina slice method for distributional solutions to elliptic equations with drift term, in preparation.

[17] T. Del Vecchio and M.M. Porzio, Existence results for a class of non-coercive Dirichlet problems. Ric. Mat. 44 (1995) $421-438$. 
[18] T. Del Vecchio and M.R. Posteraro, Existence and regularity results for nonlinear elliptic equations with measure data. Adv. Differ. Equ. 1 (1996) 899-917.

[19] G. Duro and E. Zuazua, Large time behavior for convection-diffusion equations in RN with periodic coefficients. J. Differ. Equ. 167 (2000) 275-315.

[20] G. Duro and E. Zuazua, Large time behavior for convection-diffusion equations in RN with asymptotically constant diffusion. C. R. Acad. Sci. Paris Sér. I Math. 321 (1995) 1419-1424.

[21] M. Escobedo and E. Zuazua, Long-time behaviour of diffusion waves for a viscous system of conservation laws in $\mathbb{R}^{N}$. Asymptot. Anal. 20 (1999) 133-173.

[22] J. Serrin, Pathological solutions of elliptic differential equations. Ann. Scuola Norm. Sup. Pisa Cl. Sci. 18 (1964) $385-387$.

[23] G. Stampacchia: Le problème de Dirichlet pour les équations elliptiques du second ordre à coefficients discontinus. Ann. Inst. Fourier (Grenoble) 15 (1965) 189-258. 